\title{
IMPLEMENTING AN IOT APPROACH TO CARE- AWARENESS IN THE HOME.
}

\author{
Leonidas Bourikas ${ }^{1 *}$, Philip Turner', AbuBakr Bahaj', Stephanie Gauthier ${ }^{1}$, Patrick \\ James ${ }^{1}$, Hannah Dalton ${ }^{2}$, Nick Allott ${ }^{2}$ \\ ${ }^{1}$ Energy and Climate Change Division, Sustainable Energy Research Group, (www.energy.soton.ac.uk), Faculty \\ of Engineering and Physical Sciences, University of Southampton, Southampton SO17 1BJ, UK. \\ ${ }^{2}$ NquiringMinds, Southampton Science Park, Southampton, UK. \\ *L.Bourikas@soton.ac.uk
}

Keywords: care at home, care app, independent living, occupancy behaviour, activity profile

\begin{abstract}
There is a need for new thinking in care previsions and IOT is likely to play a major role in achieving transformation of care services at lower cost. Such an approach sits at the heart of this work. Here we present the outcome of an ongoing research programme based on sensor and App interventions (trial) at homes of participants who live independently but require care. The results indicate contrasting views of carers and the persons who receive care regarding (a) the uptake and utilisation of IoT, and (b) the potential impact on the quality of life and independence of both groups. In contrast to current telecare solutions that respond to "hard"- acute alerts, our research proposes the introduction of "soft" warnings, based on the continuous evaluation of the occupancy behaviour, care conditions and the home environment. In this context the results suggest that the care needs of a person can be more important determinants of occupancy behaviour than socio-demographic attributes that are typically used in residential occupancy classification. This research under the CareTeam programme, is a non-intrusive IoT approach geared to generate proactive care-awareness to help people who receive care stay independent and active for longer.
\end{abstract}

\section{Introduction}

In 2016, around 2.2 million adults received informal and unpaid care in the UK. On average, each adult received 3,500 hours of unpaid care with an annual cost of around $£ 30,000$. Such support represents around $£ 59.5$ billion in value to the economy [1]. In 2017/2018, local authorities (LA) spent over $£ 18$ billion on adult social care [2] with around 1.8 million new clients requesting support [2]. The combination of population demographics, local authorities' budget restrictions, large wage costs for care providers and low fee rates by state-funded care contributes to the ever increasing pressure on funding in the care sector [3]. Projections by Local Government Association indicate that by $2019 / 2020$ the social care funding gap will be over $£ 1.5$ billion and projected to increase in the future [4]. With regard to informal care, $90 \%$ of the total unpaid care hours is attributed to people requiring continuous daily care (168 hours a week) [1]. This shows magnitude of the problem highlighting the reliance of some people on their carers and the responsibility that informal carers bear every day on behalf of our society.

Assisted living technology (AT) has been presented as part of a solution that will help ageing people remain active and independent in their homes for longer. Telecare and telehealth services are commonly used to remotely monitor the health of people who receive care and respond to acute events such as falls. Despite their benefits, AT solutions are often portrayed as intrusive and isolating [5] [6]; i.e. a technology that replaces human contact and monitors spaces that are private in people's homes. In the context of adult care, there are currently numerous approaches to assistive technology usage to support and improve the quality of daily life for those in care [7]. With applications ranging from cognitive orthotics [8] [9], ADL and sleep monitoring [10], detecting falls and hazards [11] and general wellbeing enhancement [12].

The Adult Support Digital Platform (ASDP), CareTeam for short, collaborative research between NQuiringMinds (IoT innovators), the University of Southampton, and Southampton City Council [13]. CareTeam is a digital platform that comprises a mobile app and a set of sensors (Fig. 1) developed in response to the challenges that adult social care present in the UK today.

This paper presents the methodology and the results of an ongoing trial of the CareTeam programme, addressing people who receive care at home and their carers' activities. The objectives of the research are:

1. Demonstrate the impact of the implementation of IoT in care at home and how this can extend independence both for the cared for and their carers.

2. Evaluate the use of sensors and their metadata for passive monitoring of background activities and environmental conditions at the home of a person who receives care.

3. Investigate opportunities for the generation of proactive, preventive alerts through tracking deviations from regular behaviour patterns of a person who receives care. Identifying a participant's activity in the home is the more complex part of the process [14] with one of the main problems being the multitude of activities that one can perform. Research into basic activities has found it possible to detect activities such as waking up and using the toilet, irregular 
activities such as leaving the house or showering are harder to predict due to their inherent randomness [15].
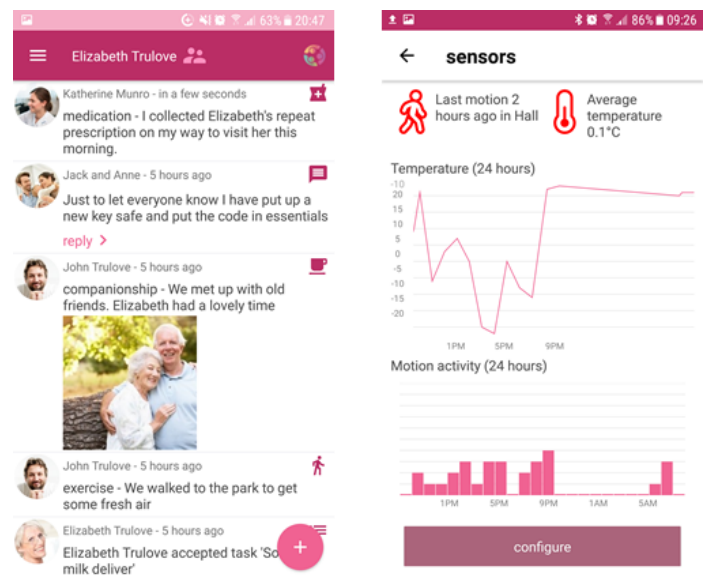

Fig. 1 Example of CareTeam app interface, comprising communication features and IoT sensors to achieve careawareness in the home.

The participants in the trial are adults who receive care at home and their carers. The CareTeam app and sensors aim to create a "safety net", a care network around the person who receives care and utilise - the family, friends and neighbours - informal carers resource. The app provides the space for the "defined and approved" members to interact in real time and catch-up with care tasks offline. Within the app, simple panels / windows have been designed for easy access, fast edit of information and selection from lists with typical events/requests. In addition, the app features enhanced safety, security and privacy choices. PIR motion sensors offer another layer of information that is trying to establish the "expected" occupancy behaviour and give a proactive and preventive character to the system based on baseline activity tracking and projections.

In the following sections, we introduce the research design or methodology, results and discussion. Finally practical implications of future IoT approach to care are reviewed and conclusions are drawn.

\section{Research design}

The research applies a mixed method approach. Objective and subjective, qualitative and quantitative data are collected and analysed. All ethical approvals were obtained including data sharing and security requirements. When the sensors were installed in the participants' homes, a short questionnaire took place to establish household and building characteristics and to estimate expected activity at different times and locations in the house. These data provided valuable information about the activity levels of the person who received care and were used in the interpretation of the activity profiles.

The functionality of the CareTeam platform and the impact of IoT on the life of the care networks were periodically assessed with feedback questionnaires, interviews and focus groups. There were both formative and summative assessments where the participants were asked to describe the use of the system, discuss any problems, give feedback and evaluate the impact CareTeam had on their everyday life.
The feedback questionnaire consisted of three parts. The first part enquires about the CareTeam users' general views on care provision, their care needs and the resources they have available. The second part reviews the impact of IoT on their quality of life (QoL). This part also addressed everyday activities, social relationships and participations, loneliness, independence and control over daily life. The third part collected feedback on the usability of the CareTeam platform with open-ended questions.

While QoL is largely determined by one's capability to be autonomous and independent [16] there is no consensus on the conceptual definition or measurement [17]. Multiple tools have been developed including the Adult Social Care Outcomes Toolkit (ASCOT) [17][18], World Health Organization Quality of Life (WHOQOL) [20], Physical Selfmaintenance Scale (PSMS) and Instrumental Activities of Daily Living Scale (IADL) [21], Older People's Quality of Life questionnaire (OPQOL) [22] and the Index of Wellbeing in Later Life [23]. For the purposes of this study, a combined questionnaire approach was chosen that included factors from these indexes relevant to the aim of the study but excluded factors such as safety and health.

As part of the CareTeam IoT approach to care, two passive infrared (PIR) motion sensors [24] were installed at the lounge and the hallway of the houses in the trial (Fig. 2). The selection of these rooms was the result of a combination of privacy issues, high likelihood of movement and activity expectations. The activity in these rooms was expected to be representative of the daytime occupancy (i.e. lounge) and the night-time activity (i.e. hallway that connects bedrooms with toilet and kitchen).
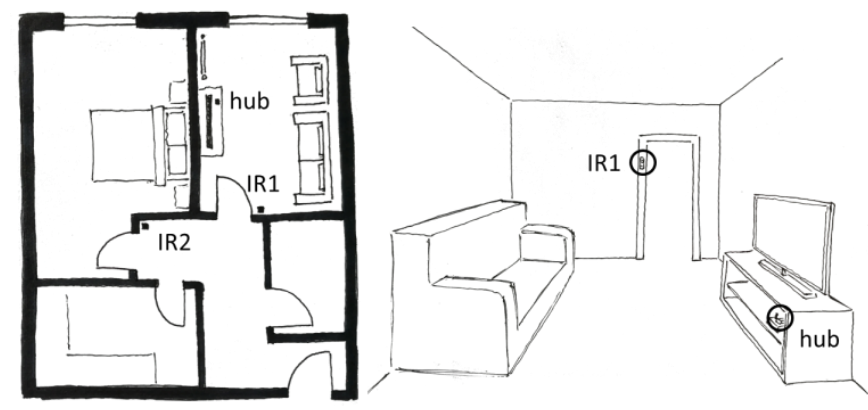

Fig. 2 Sketches showing the typical location of hub and PIR motion sensors (IR1 \&IR2) installed.

The PIR sensors (Fig. 3) have 12 meters range and a $102^{\circ} \mathrm{x}$ 92。 field of view. The PIR sensors also monitor the participants' home indoor environmental conditions, air temperature $(\mathrm{T})$ and relative humidity $(\mathrm{RH})$ are logged at a frequency of 5-minute intervals. The temperature accuracy according to the manufacturer is $+/-0.5{ }^{\circ} \mathrm{C}$ and the $\mathrm{RH}$ accuracy is +/-2 RH points [25].

The app displays the latest sensors' readings and the users can configure custom alerts on movements' frequency and temperature thresholds.

Three data processing steps were undertaken:

1. Raw data from the sensors were evaluated against the user settings for movement and temperature set points (human in-loop approach).

2. Investigation of similar patterns between groups with common characteristics, i.e. "Typical" occupancy 
behaviour profiles for groups of people with similar care requirements.

3. Trend analysis and forecasts to develop an adaptive activity baseline and track changes to the expected patterns (human out of loop).

The combination of these three processes or steps are aimed at enabling the generation of proactive warnings and help the carers assess changes in short and long term monitored behaviours.

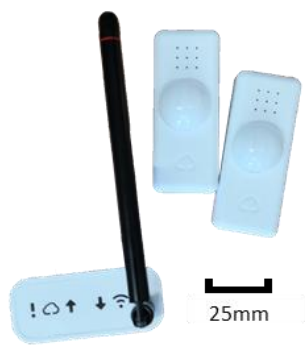

Fig. 3 The wireless PIR sensors [25] used in the study and the network interface controller (hub).

\section{Results}

The results included in this paper addressed the survey feedback from the latest three months period of the trial (October to December 2018). The monitoring results are based on the analysis of observations collected from the houses of participants during the period of a month in December 2018.

\subsection{Environmental monitoring}

Temperature observations from the PIR sensors (Fig. 4) were used to evaluate if there were "events" when the temperature exceeded $25^{\circ} \mathrm{C}$ or fell below $20^{\circ} \mathrm{C}$. The temperature range from $20^{\circ} \mathrm{C}$ to $25^{\circ} \mathrm{C}$ was considered an acceptable comfort band [26].

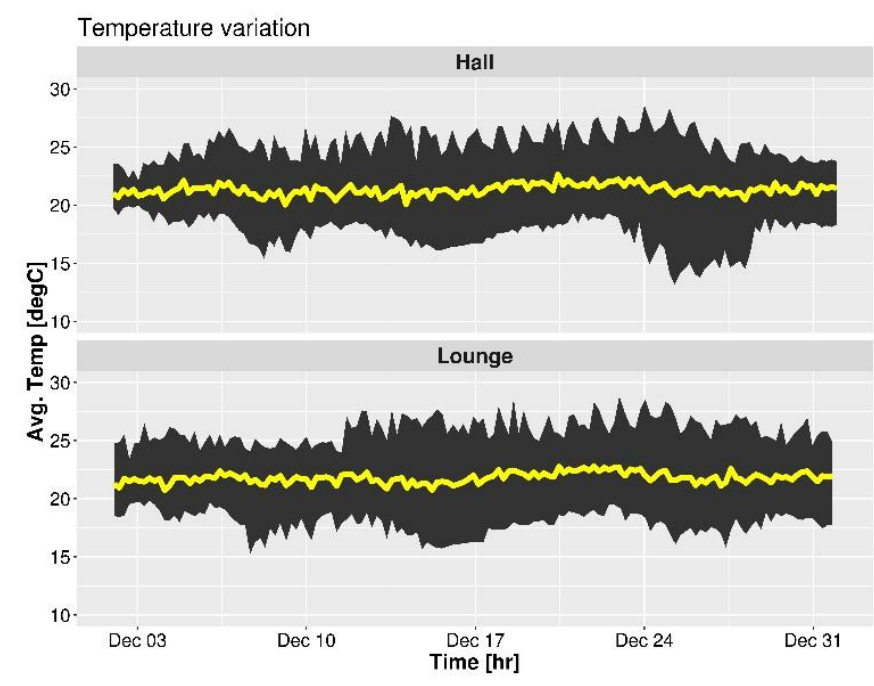

Fig. 4 Hourly average air temperature range in the hall (top) and the lounge (bottom) during December 2018. The yellow line shows the average temperature from all houses in the trial. Figure 4 shows the hourly average temperature from the homes in the trial during December 2018.

The grey ribbon shows the range of the measurements and the yellow line is the average temperature from all the houses in the trial.
The recorded temperatures indicate several temperature related "events" when warnings were sent to care networks in the app. These warnings can be very useful in the case of elderly people or people with memory loss that would otherwise not interact with the heating system.

Movement detection was used to create alerts about absence of movement for time periods longer than the ones specified by the users. The PIRs logged when movement was detected and when the status defaulted back to "timed out", usually set to reset 5 minutes after the last movement detection (i.e. five minutes without movement while status is still "movement detected"). The hourly activity profiles per household were evaluated to examine if there is a diurnal variation and any prevailing occupancy patterns. The total hourly activity is shown in Fig. 5 for the period of a month in December 2018. The different colours represent the days of a week.

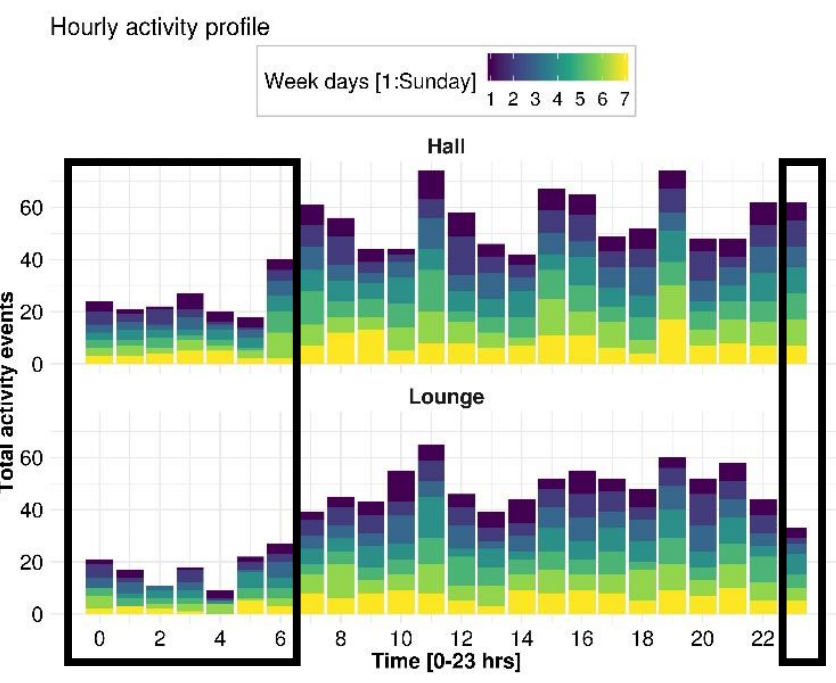

Fig. 5 Total hourly activity detected in hall (top) and lounge (bottom) for each day during December 2018. The black box marks the night-time activity cluster.

There are two distinct activity clusters identified: 1) a nighttime cluster (N) from 23:00 to 06:00 (black box in Fig. 5) and 2) a daytime cluster (D) from 07:00 to $22: 00$. The detected movement in the daytime cluster is more than the night-time in both the hallway and the lounge as expected. Saturdays (shown with yellow, 7 in Fig. 5) have increased activity during the late afternoon and early evening hours. Interestingly, Sunday and Monday (top two dark blue and blue bars respectively in Fig. 5) have a similar hourly profile.

In the early morning hours the overall activity is less but there is more activity in the hallway than the lounge. This result justifies the selection of the hallway as a location to monitor and shows that the PIR sensors detected night movements, most probably to the toilet. Most importantly, the hourly and daily profiles point out the differences and the randomness in the occupancy profiles of households with persons who receive care. The occupancy is continuous and there are not significant peaks in morning and afternoon with long periods of inactivity during the daytime as it is typical for occupancy patterns used in building energy performance simulations [27]. A likely explanation is that the occupancy behaviour of households with people who receive care is largely dictated by 
the persons' care needs and their dependence to their carers for everyday activities.

The two clusters (N \& D) were used for the calculation of the cumulative distribution function (CDF). Fig. 6 shows the probability of the hourly activity detected being less than or equal to a number of movement detection events at the monitored spaces. Each line represents a household. The movement observations were collected during the period of a month in December 2018.

The daytime cluster has higher probability to have more than three activity detections than the night-time. The lounge movement probability varies between the households. However, about half of the households have less than or equal to three movement events per hour. Two clusters of households can be identified by the daytime activity expectations in the lounge (Fig. 6). One cluster (DL1) of households with less than or equal to 3 movement events expected per hour and a second cluster (DL2) where the expected occupancy is above the three events and less than or equal to five movement events.

The questionnaire data were examined to identify if there were any attributes that could justify the difference in daytime activity between clusters DL1 and DL2. This review shows that most of the people who receive care in the households reported mobility difficulties and almost continuous occupancy of the lounge during the daytime period. Interestingly, the two clusters, DL1 and DL2, comprise of households of different size and different house types. In DL1 in particular, the households have at least 2 occupants and most of them live in 2 floor houses. On the other hand, households in DL2 have a single occupant and they are flats.

(A) Cumulative distribution of hourly activity [0700-2200]

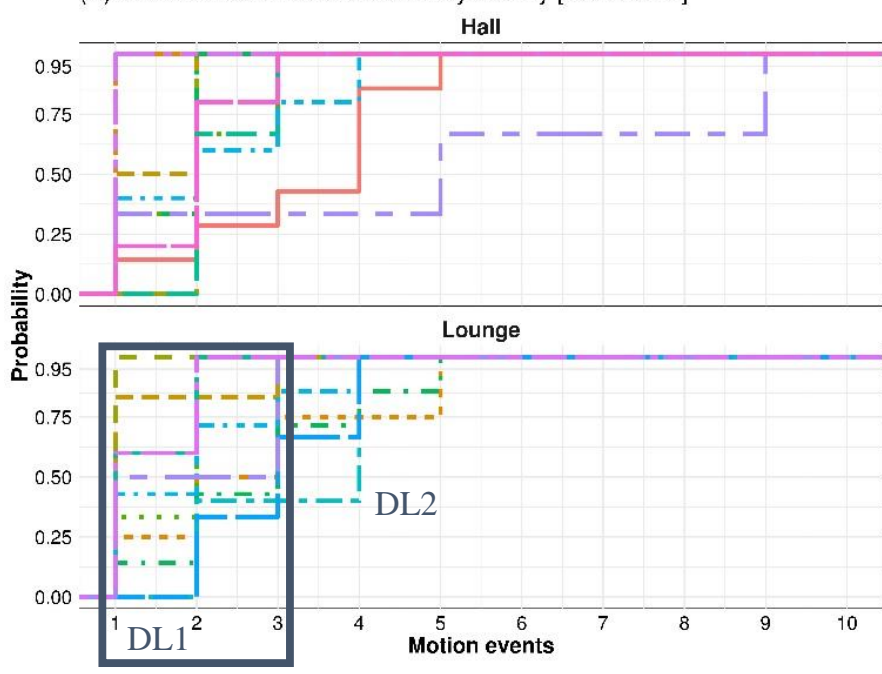

Fig. 6 Probability of day-time movement in hall (top) and lounge (bottom) based on PIR motion observations in December 2018. DL1 (grey box) and DL2 are two clusters according to the activity levels. Each line represents a household in the trial.

This result reinforces the idea that traditional occupancy profiling approaches often do not apply in the case of care at home. In this case, the smaller size of the houses in DL2 might be the reason for spending more time in the lounge and not in other rooms.

It is difficult to associate the daytime movement observations with specific care attributes from the emerging clusters. The night-time movement (Fig. 7), especially in the hall area, is highly likely to be associated with sleeping difficulties and visits to the toilet. This is better shown in Fig. 7 that focuses on the probability of activity detected between 01:00 and 05:00. In fact, two sub-clusters were identified that had 1 to 2 activity events (NH1) and 3 to 5 events (NH2) respectively. The main differentiator between $\mathrm{NH} 1$ and $\mathrm{NH} 2$ was a difference to the Activities for Daily Living (ADL) [21] assessment scores.

\section{(B) Cumulative distribution of hourly activity [0100-0500]}

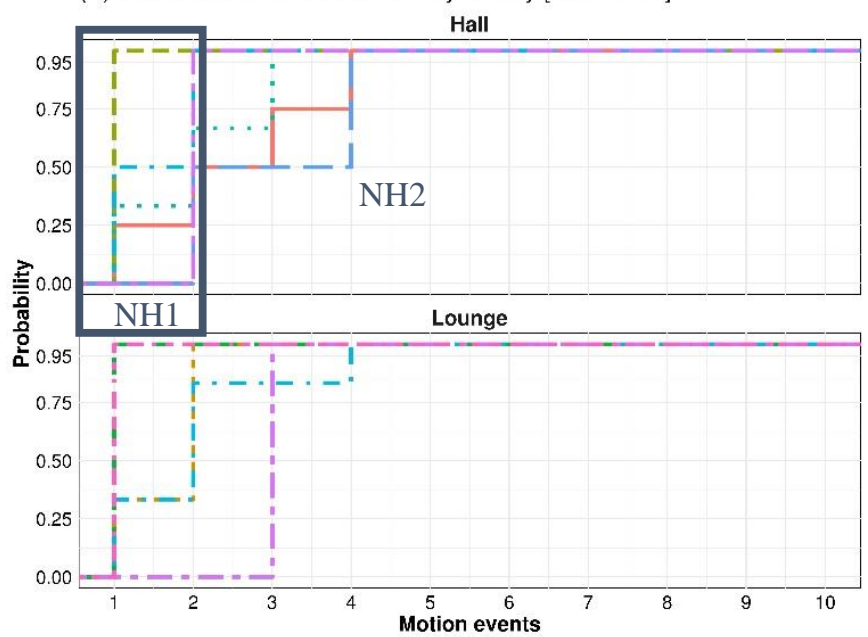

Fig. 7 Probability of night-time movement in hall (top) and lounge (bottom) based on PIR motion observations in December 2018. NH1 (grey box) and NH2 are two clusters according to the activity levels. Each line represents a household in the trial.

The ADL is commonly used as part of the assessment to determine if a person needs and qualifies for adult social care and the level of help required. It is an evaluation of the capability of a person to undertake independently activities such as laundry, handling money, bathing, going in the toilet etc. High score is associated with high care requirements. NH2 that had the highest probability to have 3 to 5 movement events per hour had low scores in ADL whereas the NH1 group had high scores and most of the people were receiving support with common everyday tasks.

The cluster with the ADL high scores was further analysed to develop a representative, "baseline" occupancy behaviour profile. The main hypothesis was that the ADL assessment criteria (i.e. care needs) can be grouped and linked directly with expected activity patterns. That refinement would produce a set of care typologies in relation to the monitored occupancy behaviour. At this stage of the study however, the size of the sample and the duration of the observations did not allow for a meaningful clustering according to specific care requirements groups. 


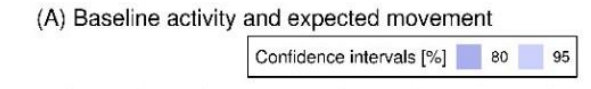

2.0

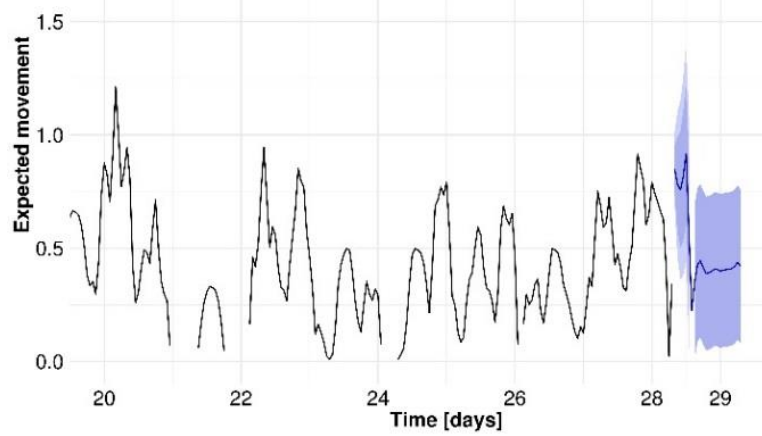

(B) Baseline activity and expected movement

2.0

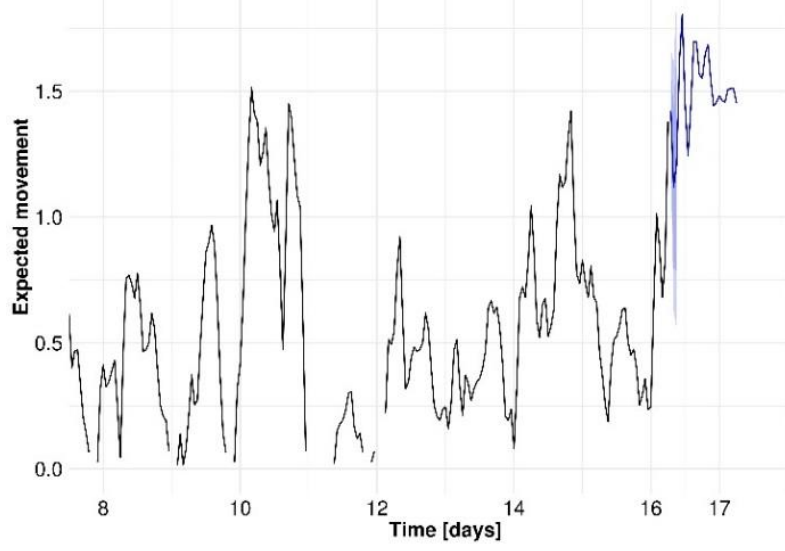

(C) Baseline activity and expected movement

2.0

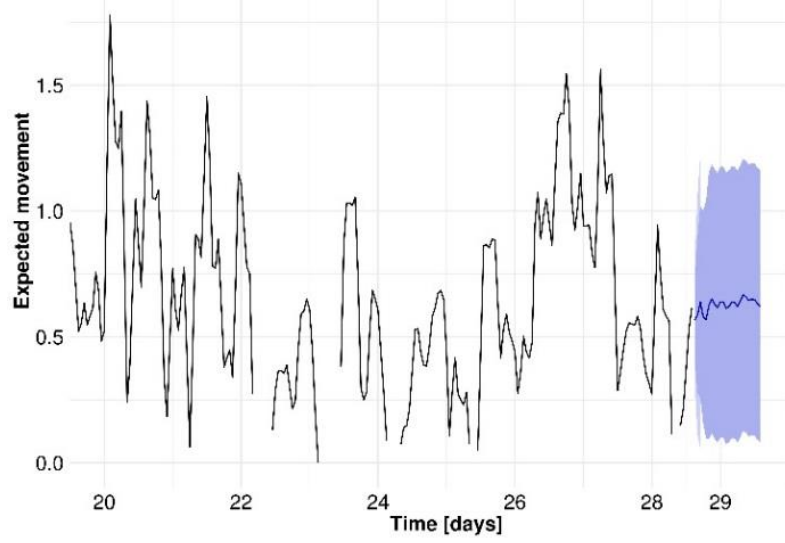

Fig. 8 Baseline activity (black line) and $24 \mathrm{hr}$ forecasts of expected movement (blue line, CI 80\% and 95\%). A,B,C refer to different participants in the NH1 - ADL high score cluster. Dates shown are in December 2018.

Fig. 8 shows the smoothed baseline activity (moving average of $6^{\text {th }}$ order) in autoregressive integrated moving average ARIMA models for three participants in the NH1 cluster with ADL high scores. The models were used to forecast the movement for the next 24 hours based on the past observations. These ARIMA models are a first effort to create short-term projections for the baseline, expected activity. The results would be more meaningful if interpreted in the context of probability of activity at specific hours of day. These person specific models allow the tracking of deviations from the baseline profile and they can be used to identify changes in the expected activity projections. The retraining of the model with current observations can develop an adaptive baseline that could be used to create proactive warnings about changes that cannot be explained by the occupancy behaviour profiles.

\subsection{Surveys and feedback}

Based on the feedback interviews and the questionnaire responses, about half of the participants "disagree" and "strongly disagree" that they have the information they need to make care choices. Whereas the majority "disagree" that the professionals involved with their care talk to each other and that their informal carers' are recognised and supported. These results support this study's initial evidence and rationale regarding the value of informal, unpaid care and the stress of the carers. The results also show that there is a problem with the coordination of care and how the persons in care and their families perceive formal support.

Evidence from literature suggest that most of the carers are keen to adopt an IoT approach often wishing for surveillance to provide reassurances, and are unconcerned with the potential intrusion and stigmatisation for the cared for having a constant reminder of their current state [5]. This was found to be the case during the recruitment process with carers often keen to sign up but after conversing with the person cared for having to pull-out in order to respect their privacy wishes. In this case, the persons who receive care rejected the implementation of IoT approaches because they perceive the sensors as constant surveillance devices and a sacrifice of their privacy independence. That is exactly the opposite effect of this research's intentions and aim.

All the users seem to agree that the use of the CareTeam platform has not affected their wellbeing and in particular there was not any impact reported on the feelings of loneliness and being active in the community. In fact there is evidence in the literature showing that increased use of Information and Communication Technology (ICT) could have adverse effects on isolation, whereby it reinforces existing social networks but does not build new ones [28].

The questionnaires have also indicated that both the carers and the persons in care prefer very simple app features and the existence of many functions is likely to be a deterrent on the use of the app. This was a recurrent theme in the feedback with most of the users rating the usability of the app as "fairly" or "very difficult" despite the fact that training was offered to all the users. The feedback showed that the users are mostly interested in the "chat", communication function and the sensors whereas the event planning and calendar arrangements features were not used broadly.

In the interviews it became apparent that although the majority of the persons who receive care had access to a mobile phone device, a large number of them would use it only as a telephone and disregard the use of apps. The feedback can be explained to some extent by the sample demographics with most of the participants being older than 65 years. This is still valid 
feedback as the majority of IoT users in the context of care are likely to be part of this demographic group.

\section{Conclusion}

The feedback during the ongoing CareTeam very small trial highlight the lack of coordination of care. This has a large impact on a person's care and their families' views and expectations for formal support. In the near future, IoT will have prominent role to play in the coordination of care to achieve increasing care efficiency and communication between formal and unpaid care providers. However, this trial although small in sample size, concludes that the IoT design concepts may fail to take into account the needs and skills of the users. In particular, care professionals and those who receive care, all seem to prefer very simple app features and targeted functions that can help them accomplish predefined tasks with less effort. This aspect may be critical to IoT implementation in care provision as the users of the technology are not aspired by the modernity, and technical specifications but they are mostly attracted by the trustworthiness and inclusivity features.

Several informal carers and care organisations were interested in the CareTeam background sensing capabilities and the proactive warnings based on household specific occupancy profiles. Commonly used occupancy patterns are based on group categorisations according to the household composition and the working status [27]. However, the initial findings from this study suggest that in the context of adult care, the care needs of the person who receive care and their independence from carers can be more important determinants of occupancy than generic socio-demographic attributes. Interestingly, during night-time, the activity levels indicated that the occupancy is related to the care needs of the participants in the trial with those having higher care needs showing less activity than the cluster with the low care requirements.

This study evaluated the use of household specific ARIMA models for the development of baseline occupancy profiles from PIR motion observations. Short-term projections were successfully created to establish an "expectation" of activity at specific times. The study data collection highlighted challenges in participants' uptake. Furthermore the data analysis revealed false-positive readings due to pets and visitors [29], also the variation in the sensitivity of the PIR sensors needed further calibration.

Future work will cover larger sample size and will aim to integrate a probabilistic approach to the occupancy profiles and evaluate the merits of different machine learning approaches in their use with occupancy related time series in the context of care at home. CareTeam aspires to introduce a new paradigm for care coordination and the use of IoT for the development of person specific, adaptive and evolving background data interpretation and proactive care-awareness to support independent living.

\section{Acknowledgements}

This work is part of the activities of the Energy and Climate Change Division and the Sustainable Energy Research Group at the University of Southampton (www.energy.soton.ac.uk). This work is also in partnership with NquiringMinds (https://nquiringminds.com/) and Southampton City Council, Southampton, UK. The work is supported by an Innovate UK Grant number TS/P013163/1.

\section{References}

[1] Office for National Statistics, "Household satellite account, UK : 2015 and 2016," no. October, pp. 1-19, 2018.

[2] Adult Social Care Statistics and NHS Digital, "Adult Social Care Activity and Finance Report, England 2017-2018," no. October, pp. 152, 2018.

[3] Competition \& Markets Authority, "Care homes market study: summary of final report," 2017.

[4] H. Cromarty, "Adult Social Care Funding ( England ). Briefing paper CBP07903.," no. October 2018, 2018.

[5] S. Stowe and S. Harding, "Telecare, telehealth and telemedicine," Eur. Geriatr. Med, vol. 1, no. 3, pp. 193-197, 2010.

[6] C. Sanders et al., "Exploring barriers to participation and adoption of telehealth and telecare within the Whole System Demonstrator trial: A qualitative study," BMC Health Serv. Res., vol. 12, no. 1, p. 220, 2012

[7] J. C. Augusto, "AAmbient Intelligence : The Confluence of Ubiquitous / Pervasive Computing and Artificial Intelligence," in Intelligent Computing Everywhere, A. J. Schuster, Ed. London: Springer, 2007, pomputing $213-234$

[8] I. Qudah, P. Leijdekkers, and V. Gay, "Using mobile phones to improve medication compliance and awareness for cardiac patients," Proc. 3rd Int. Conf. PErvasive Technol. Relat. to Assist. Environ. - PETRA '10, p. $1,2010$.

[9] D. U. Khan, K. A. Siek, J. Meyers, L. M. Haverhals, S. Cali, and S. E. Ross, "Designing a personal health application for older adults to manage medications," Proc. ACM Int. Conf. Heal. informatics - IHI '10, p. 849, 2010

[10]M. E. Pollack et al., "Autominder: An intelligent cognitive orthotic system for people with memory impairment," Rob. Auton. Syst., vol. 44, no. 3-4, pp. 273-282, 2003.

[11]W. Lee and X. Qin, Lecture Notes in Computer Science. 2003.

[12]P. Rashidi and A. Mihailidis, "A Survey on Ambient-Assisted Living Tools for Older Adults," vol. 17, no. 3, pp. 579-590, 2013.

[13]NquiringMinds, University of Southampton, and Southampton City Council, "CareTeam - Adult Support Digital Platform. CareTeam is a research programme partly funded by Innovate UK. CareTeam is a nonintrusive IoT approach geared to generate proactive care-awareness to help people who receive care stay independent and active." [Online]. Available: http://www.energy.soton.ac.uk/careteam-adult-supportdigital-platform/. [Accessed: 25-Feb-2019].

[14]L. Chen, J. Hoey, C. D. Nugent, D. J. Cook, and Z. Yu, "Sensor-based activity recognition," IEEE Trans. Syst. Man Cybern. Part C Appl. Rev., vol. 42, no. 6, pp. 790-808, 2012

[15]M. T. Moutacalli, A. Bouzouane, and B. Bouchard, "The behavioral profiling based on times series forecasting for smart homes assistance," J. Ambient Intell. Humaniz. Comput., vol. 6, no. 5, pp. 647-659, 2015.

[16]F. Jacobsen, “Active ageing," Int. Pract. Dev. J., vol. 7, no. Suppl, pp $1-13,2017$.

[17]A. Bowling, Ageing Well. Quality of Life in Old Age. Maidenhead, UK: Open University Press, 2005.

[18]A. Netten et al., "Outcomes of social care for adults: Developing a preference-weighted measure," Health Technol. Assess. (Rockv)., vol. 16, no. 16, pp. 1-165, 2012.

[19]J. Caiels, R. Masrani, J. Forder, A. Netten, and K. Windle, "Measuring the outcomes of low-level services: Final report," no. June, 2010.

[20]The WHOQOL-Group, "The development of the World Health Organization quality of life assessment: International perspectives," pp. 41-57, 1998

[21]M. P. Lawton and E. M. Brody, "Assessment of older people: selfmaintaining and instrumental activities of daily living.," Gerontologist, vol. 9 , no. 3, pp. 179-186, 1969.

[22]A. Bowling, "The Psychometric Properties of the Older People's Quality of Life Questionnaire, Compared with the CASP-19 and the WHOQOL-OLD," Curr. Gerontol. Geriatr. Res., vol. 2009, pp. 1-12, 2009

[23]M. Green, J. Iparraguirre, S. Davidson, and A. Zaidi, "A summary of Age UK's Index of Wellbeing in Later Life," pp. 1-16, 2017.

[24]CAO Gadgets LLC, "Wireless Sensor Tags." [Online]. Available: http://wirelesstag.net/. [Accessed: 19-Feb-2019].

[25]CAO Gadgets LLC, "Wireless Sensor Tags."

[26]R. Amin, D. Teli, P. James, and L. Bourikas, "The influence of a student's 'home' climate on room temperature and indoor environmental controls use in a modern halls of residence," Energy Build., vol. 119, pp. 331-339, 2016.

[27] V. Aragon, S. Gauthier, P. Warren, P. A. B. James, and B. Anderson, "Developing English domestic occupancy profiles," Build. Res. Inf., vol. 47, no. 4, pp. 375-393, 2019

[28]J. Damant, M. Knapp, P. Freddolino, and D. Lombard, "Effects of digital engagement on the quality of life of older people," Heal. Soc. Care Community, vol. 25, no. 6, pp. 1679-1703, 2017.

[29]C. Spataru and S. Gauthier, "How to monitor people 'smartly' to help reducing energy consumption in buildings?," Archit. Eng. Des. Manag., vol. 10, no. 1-2, pp. 60-78, 2014. 